\title{
A Case Report of Rare Acute Ischemic Stroke Due to Meningeal Irritation
}

\author{
Reem AlSherooqi1 ${ }^{1}$ Jad Alkhoury1, Moiz Bakhiet ${ }^{1,2 *}$ \\ ${ }^{1}$ Department of Internal Medicine, King Hamad University Hospital Princess Al-Jawhara Center for Genetics and Inherited \\ Diseases, Arabian Gulf University, Manama, Bahrain \\ ${ }^{2}$ Department of Molecular Medicine, College of Medicine and Medical Sciences, Princess Al-Jawhara Center for Genetics and \\ Inherited Diseases, Arabian Gulf University, Manama, Bahrain \\ Email: *moiz@agu.edmoiz@agu.edu.bhu.bh
}

How to cite this paper: AlSherooqi, R. Alkhoury, J. and Bakhiet, M. (2018) A Case Report of Rare Acute Ischemic Stroke Due to Meningeal Irritation. World Journal of Neuroscience, 8, 171-177. https://doi.org/10.4236/wjns.2018.82015

Received: March 30, 2018

Accepted: May 4, 2018

Published: May 7, 2018

Copyright $\odot 2018$ by authors and Scientific Research Publishing Inc. This work is licensed under the Creative Commons Attribution International License (CC BY 4.0).

http://creativecommons.org/licenses/by/4.0/

\begin{abstract}
Stroke is a rapidly developing symptom or sign of loss of cerebral function secondary to interruption of the blood supply due to a thrombus or hemorrhage. These diseased vessels are usually affected by well-recognized risk factors, but in some cases other rare conditions may be related to stroke development. Herein, we introduce one of these rare stroke conditions in a 37 -year-old previously healthy male who presented to the emergency department with misleading symptoms and signs of reduced level of consciousness, isolated medical $3^{\text {rd }}$ nerve palsy and possible drug overdose. A thorough neurological examination and investigation demonstrated a case of acute ischemic stroke as a consequence of meningeal irritation with an underlying inflammatory response. The patient was treated simultaneously for both conditions and responded dramatically with full recovery upon discharge. Thus, consideration of acute stroke in patients with no previous known risk factors is highly recommended especially with unusual neurological findings.
\end{abstract}

\section{Keywords}

Meningitis, Weakness, Diplopia, Photophobia, EEG

\section{Introduction}

Stroke is recognized as the third leading cause of death worldwide and is associated with permanent disability [1]. Traditional vascular risk factors affecting blood vessels and circulation are the most common causes of stroke. These factors are common in young adults with ischemic stroke, which include Dyslipidemia, smoking, Hypertension, Patent Foramen Ovale, large-artery atherosclerosis, small-vessel occlusion and cardioembolism [2]. 
There are many less common causes of stroke which can be sub classified into inflammatory and non-inflammatory vessel disorders, hematological disorders, prothrombotic (clotting) disorders, Migraine-Related Stroke and Cerebral Venous Thrombosis (CVT). Examples of non-inflammatory blood vessel diseases include Fibromuscular Dysplasia-a disorder that results in an abnormal development of cells in the wall of arteries [3], Reversible Cerebral Vasoconstriction Syndromes [4], radiation therapy for cancers of the head and neck [5], Moyamoya disease-a rare disorder causing blocked arteries in the basal ganglia [6], Fabry Disease-an X-linked rare genetic disorder causing lipids to deposit in the internal lining of blood vessels [7]. On the other hand, the non-infectious and inflammatory vasculitides that lead to stroke formation comprise Takayasu disorder, Giant Cell Arteritis, Churg-Strauss vasculitis, Wegener's Granulomatosis, Polyarteritis Nodosa, Buerger's disease, Microscopic Polyangitis, Amyloid- $\beta$-related Angiitis, and isolated vasculitis of the central nervous system (CNS) [8].

Ischemic stroke may also be associated with several blood coagulopathies affecting the red blood cells, platelet function, clotting factors or fibrinolysis [9]. Antiphospholipid Antibody Syndrome and Sickle Cell Disease may also be associated with the development of ischemic stroke [10] [11]. There is evidence that migraine patients especially those with aura, may develop stroke through an unknown mechanism. Nevertheless, migraine was also found to be associated with platelet abnormalities and clotting disorders predisposing to ischemic events [12]. Additionally, CVT is a rare cause of stroke [13].

Meningitis is the inflammation of the meninges due to bacterial, viral, or fungal infections. Other causes may include carcinoma and drugs. Diagnosis can be made clinically and biochemically through clinical symptoms including headache and photophobia as well as signs such as Kernig's and Brudzinski's signs. Detecting white blood cells (WBCs) in the CSF analysis can also aid our diagnosis. Meningitis can be classified into acute of chronic based on the onset of symptoms, CSF analysis and underlying conditions. Acute meningitis presents in hours or days [14].

This paper reports a rare case of acute ischemic stroke in a young healthy male that occurred as a consequence of meningeal irritation thus presenting with atypical signs and symptoms.

\section{Case}

A 37-year-old previously healthy male presented to the emergency room (ER) with complaints of weakness and fatigue, double vision and photophobia of few hours' duration. It was not associated with fever, vomiting, abdominal pain or any change in bowel habits. There was no chest pain, palpitations, shortness of breath, paroxysmal nocturnal dyspnea or loss of consciousness. No recent medications were started.

The patients' past medical history (PMH), past surgical history (PSH) and 
family history $(\mathrm{FH})$ were unremarkable. He was not on regular medications with no allergies and denied any history of smoking.

On examination, the patient was vitally stable and afebrile. His abdominal, respiratory and cardiovascular examinations were unremarkable. Neurological examination was difficult to assess, as patient was very drowsy. However, plantars were down going, and pupils were pinpoint bilaterally. There were no obvious needle marks and lower limb examination was normal.

Laboratory investigations (hematological and biochemical) were all within normal levels. CT Brain was also done at the level of the ER that was unremarkable.

The patient was admitted to the ward with the initial impression of reduced level of consciousness with isolated medical $3^{\text {rd }}$ nerve palsy. Drug overdose was a differential at the time of admission. Drug levels (Acetaminophen), urine toxicology and MRI (Magnetic resonance imaging) brain were requested. The patient also received a dose of Naloxone that improved his level of consciousness for a short period of time.

The Neurologist saw the patient the next day in the ward, and examination showed response to painful stimuli. He was vitally stable with neck rigidity and positive Kernig's sign. The right pupil was constricted whereas the left one was dilated. Plantars were down going bilaterally.

A differential diagnosis of absence seizure, meningitis and possible stroke was made. The patient was kept in isolation and planned for lumbar puncture (LP). He was also scheduled for an electroencephalogram (EEG), MRI brain and Carotid Doppler. After LP was done, he was started on Ceftriaxone $2 \mathrm{~g}$ once daily, Vancomycin $1600 \mathrm{mg}$ every 8 hours intravenously and Acyclovir $800 \mathrm{mg}$ every 8 hours intravenously. He was also started on Keppra $1 \mathrm{~g}$ twice daily intravenously as a prophylaxis for seizure disorder.

When patient was reviewed the next day, he was fully conscious. Laboratory investigations including complete blood count (CBC), electrolytes and calcium, C-reactive protein (CRP), hepatitis profile, Syphilis, HIV, Toxoplasmosis screen, ANA and lipid profile were normal. However, Protein $\mathrm{C}$ and $\mathrm{S}$ were elevated $->141 \%$ and $>126 \%$ respectively. CSF analysis showed elevated protein level $(80 \mathrm{mg} / \mathrm{dl})$. The gram stain showed no organism, acid-fast bacilli (AFB) negative and cultures were negative.

MRI Brain showed acute infarcts in bilateral thalamus, meningeal irritation (Figure 1) and bilateral sinusitis. There were sharp wave discharges in both temporo-occipital regions on his EEG (Figure 2). Carotid Doppler showed no stenosis or atherosclerotic plaques.

Day 3 of his admission, the patient was conscious, alert and oriented. Neck rigidity improved and Kernig's sign was negative. The patient was kept under observation for one week and was discharged in a good and stable condition.

\section{Discussion}

Per definition, meningitis is inflammation of the meninges of the CNS [14]. 


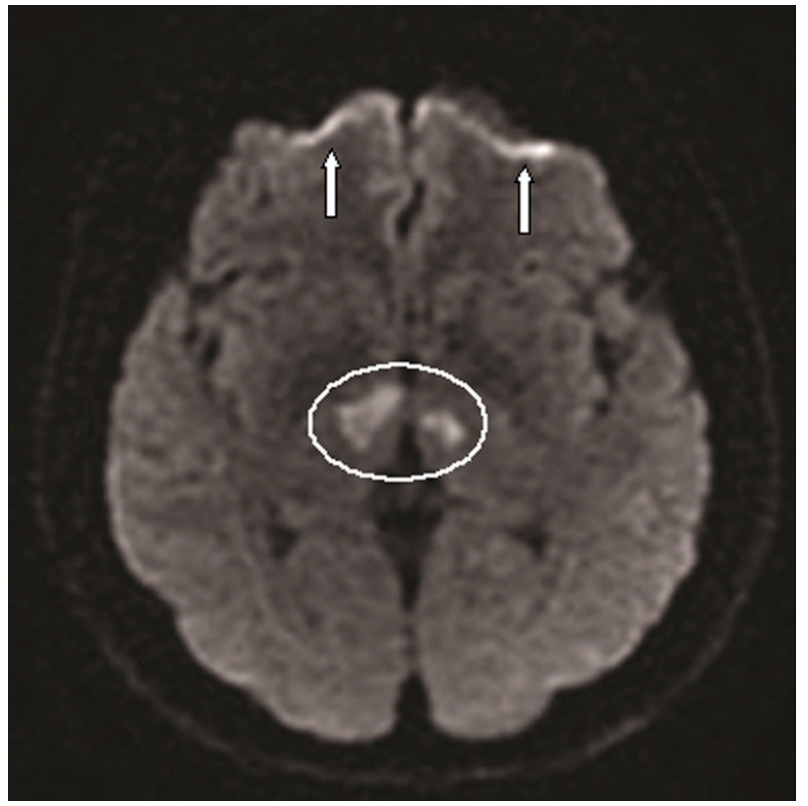

Axial Diffusion-weighted magnetic resonance imaging (ADW-MRI) revealing hyperintensities in bilateral thalamus (circle) suggesting acute infarctions, because of restricted diffusion in the corresponding sites, and probably due to the early meningitic vascular complications. Arrows depict meningeal irritation/inflammation.

Figure 1. Magnetic resonance imaging (MRI) of the brain.

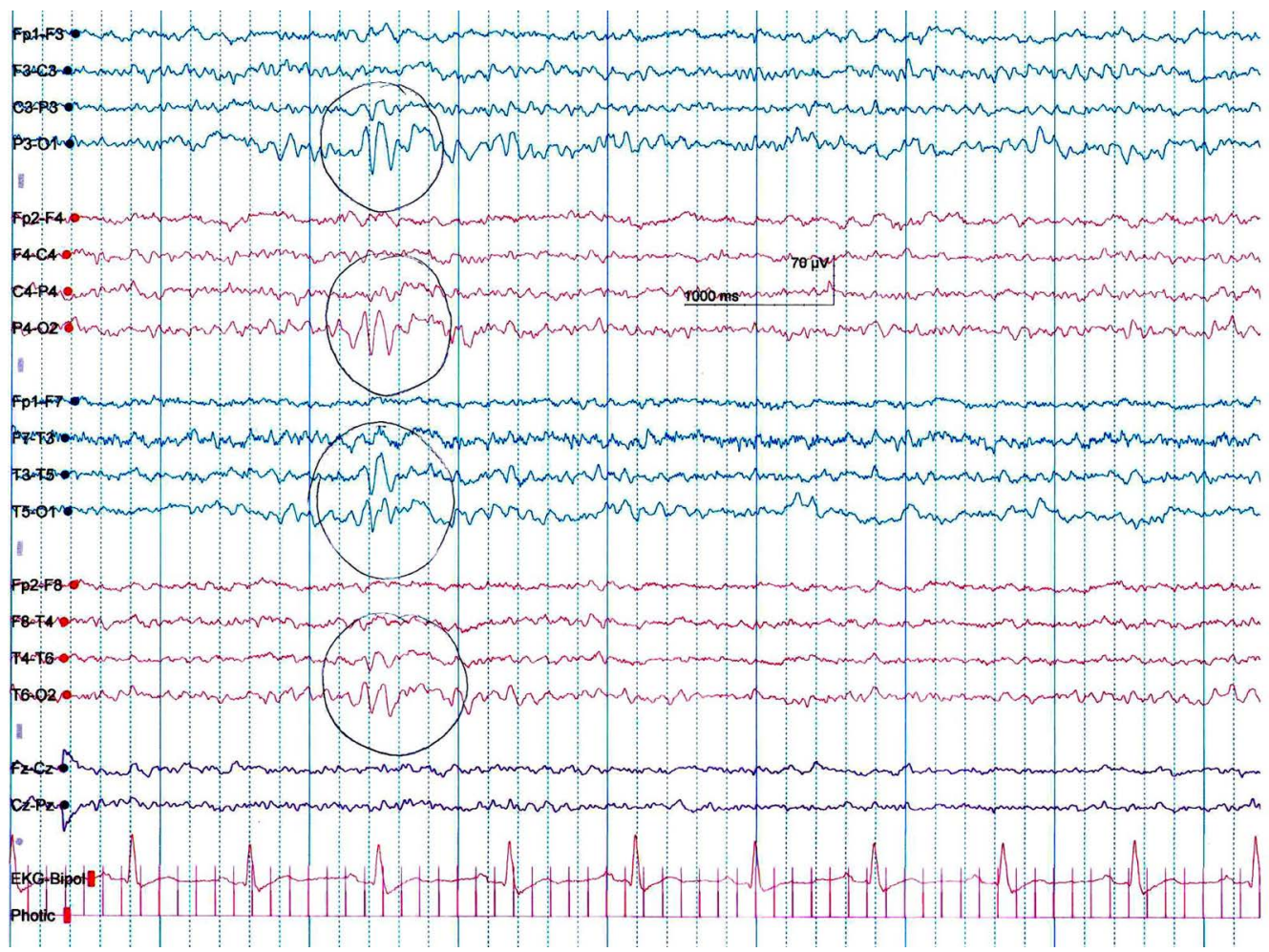

A 21 channel digital video EEG recording performed in the EEG laboratory using the standard 10 - 20 International Electrode Placement System. Hyperventilation and Photic stimulation was done. EEG was recorded in a wake stage. The posterior dominant rhythm consisted of well-organized and symmetrical $8-9 \mathrm{~Hz}$ alpha activity predominantly seen in the posterior head region. This activity was reactive to eye opening. Photic stimulation induced the background activity bilaterally. Intermittent burst of sharp wave discharges in both the temporo-occipital regions were noted.

Figure 2. Electroencephalogram (EEG). 
Stroke-as stated by the American Stroke Association (ASA) and the American Heart Association (AHA)-is a CNS (brain and/or spinal cord) infarction, or death of retinal cells as a result of ischemia, established by clinical signs of permanent damage, neuroimaging and neuropathological evidence. Ischemic stroke refers to a CNS infarction attended by obvious symptoms, whereas silent infarction produces no known symptoms [15].

The common causes of ischemic stroke are related to traditional risk factors affecting the cerebrovascular circulation. Stroke was considered as symptoms that are quickly arising or signs of nonappearance of cerebral function without obvious reasons except for a vascular cause. The case presented in this study display a young adult male who was not known to have any medical condition (especially of vascular origin) or any risk factor for stroke. The only triggering factor for his stroke was the meningeal irritation that was most probably related to an inflammatory process rather than an infection.

It has been reported previously that certain inflammatory processes of systemic origin and infectious agents can lead to the development of stroke. Ionita $\mathrm{CC}$ and colleagues have looked into different infections and their relationship to acute ischemic stroke. They demonstrated that infectious endocarditis, meningoencephalitis, and human immunodeficiency virus infections were a direct cause of stroke and concluded that infections, whether acute or chronic, can cause acute ischemic stroke. The most likely explanation is the development of enhanced immunohematologic changes and atherosclerosis [16]. However, in our case there was no infectious agent isolated and no CSF abnormality was detected to confirm bacterial or viral (aseptic) meningitis. High protein level was suggestive of an inflammatory process which might have caused the meningeal irritation. Meningitis was also evident with the clinical signs and symptoms. In addition, EEG findings (Figure 2) and meningeal irritation on MRI also supported our diagnosis (Figure 1). The cause of inflammation that resulted in the meningeal irritation is not clear to us, but it could be secondary to previous exposure to an irritating agent or an infection that has resolved and the products of that infectious agent such as endotoxin or viral protein has generated a pro-inflammatory cytokine response. It is well known that inflammation leads to activation of coagulation and that pro-inflammatory cytokines can activate the coagulation system and down-regulate important physiologic anticoagulant processes resulting in vascular complications thus leading to stroke [17]. Inflammatory vasculitis subsequent to meningeal irritation/inflammation can cause brain infarction as well [8]. The patient improved after a combination of anti-viral, anti-bacterial and anti-epileptic medications that were all required in the management and were given empirically as the underlying causative agent and mechanism were not identified.

\section{Conclusion}

Acute stroke should be considered in all age groups with no previous acknowl- 
edged risk factors affecting blood vessels and circulation among other possible differential diagnosis. Such factors may include inflammatory processes affecting the meninges and/or the brain.

\section{Acknowledgements}

We gratefully acknowledge King Hamad University Hospital for supporting this case study.

\section{Funding}

This work was supported by the Department of Internal Medicine, King Hamad University Hospital.

\section{Ethics Approval and Consent to Participate}

This is a case report of standard treatment for an unusual presentation of a disease. There was no treatment given to the patient outside the standard of care. We confirm that the case report was approved by the institute's committee on human research to be submitted for publication.

\section{Consent to Publish}

Written informed consent was obtained from the patient for his anonymized information to be published in this article.

\section{Availability of Data and Materials}

Data supporting the conclusions of this article are presented in the manuscript.

\section{Authors' Contributions}

RAS contributed to acquisition of data, analysis and interpretation of data. JA was involved in drafting the manuscript and revising it critically for important intellectual content. MB is the principle investigator (consultant) and wrote the paper.

\section{References}

[1] Donnan, G.A., Fisher, M., Macleod, M. and Davis, S.M. (2008) Stroke. Lancet, 371, 1612-1623. https://doi.org/10.1016/S0140-6736(08)60694-7

[2] Renna, R., Pilato, F., Profice, P., Della Marca, G., Broccolini, A., Morosetti, R., et al. (2014) Risk Factor and Etiology Analysis of Ischemic Stroke in Young Adult Patients. Journal of Stroke and Cerebrovascular, 23, e221-e227. https://doi.org/10.1016/j.jstrokecerebrovasdis.2013.10.008

[3] Kirton, A., Crone, M., Benseler, S., Mineyko, A., Armstrong, D., Wade, A., et al. (2013) Fibromuscular Dysplasia and Childhood Stroke. Brain, 136, 1846-1856. https://doi.org/10.1093/brain/awt111

[4] Miller, T.R., Shivashankar, R., Mossa-Basha, M. and Gandhi, D. (2015) Reversible Cerebral Vasoconstriction Syndrome, Part 2: Diagnostic Work-Up, Imaging Evaluation, and Differential Diagnosis. American Journal of Neuroradiology, 36, 1580-1588. 
https://doi.org/10.3174/ajnr.A4215

[5] Arthurs, E., Hanna, T.P., Zaza, K., Peng, Y. and Hall, S.F. (2016) Stroke after Radiation Therapy for Head and Neck Cancer: What Is the Risk? International Journal of Radiation Oncology, Biology, Physics, 96, 589-596.

https://doi.org/10.1016/j.ijrobp.2016.07.007

[6] Jang, D.K., Lee, K.S., Rha, H.K., Huh, P.W., Yang, J.H., Park, I.S., et al. (2014) Clinical and Angiographic Features and Stroke Types in Adult Moyamoya Disease. American Journal of Neuroradiology, 35, 1124-1131. https://doi.org/10.3174/ajnr.A3819

[7] Shi, Q., Chen, J., Pongmoragot, J., Lanthier, S. and Saposnik, G. (2014) Prevalence of Fabry Disease in Stroke Patients-A Systematic Review and Meta-Analysis. Journal of Stroke and Cerebrovascular Diseases, 23, 985-992.

https://doi.org/10.1016/j.jstrokecerebrovasdis.2013.08.010

[8] Adams Jr., H.P. (2014) Cerebral Vasculitis. Handbook of Clinical Neurology, 119, 475-494. https://doi.org/10.1016/B978-0-7020-4086-3.00031-X

[9] Arboix, A., Jimenez, C., Massons, J., Parra, O. and Besses, C. (2016) Hematological Disorders: A Commonly Unrecognized Cause of Acute Stroke. Expert Review of Hematology, 9, 891-901. https://doi.org/10.1080/17474086.2016.1208555

[10] Okuma, H. and Kitagawa, Y. (2013) Diagnosis and Potential Treatment of Antiphospholipid Syndrome-Related Mainly on Ischemic Stroke. Brain and Nerves, 65, 1319-1332.

[11] Talahma, M., Strbian, D. and Sundararajan, S. (2014) Sickle Cell Disease and Stroke. Stroke, 45, e98-e100. https://doi.org/10.1161/STROKEAHA.114.005144

[12] Sacco, S. and Kurth, T. (2014) Migraine and the Risk for Stroke and Cardiovascular Disease. Current Cardiology Reports, 16, 524.

https://doi.org/10.1007/s11886-014-0524-1

[13] Coutinho, J.M. (2015) Cerebral Venous Thrombosis. Journal of Thrombosis and Haemostasis, 13, S238-S244. https://doi.org/10.1111/jth.12945

[14] Putz, K., Hayani, K. and Zar, F.A. (2013) Meningitis. Prim Care, 40, 707-726. https://doi.org/10.1016/j.pop.2013.06.001

[15] Sacco, R.L., Kasner, S.E., Broderick, J.P., Caplan, L.R., Connors, J.J., Culebras, A., et al. (2013) An Updated Definition of Stroke for the 21st Century: A Statement for Healthcare Professionals from the American Heart Association/American Stroke Association. Stroke, 44, 2064-2089. https://doi.org/10.1161/STR.0b013e318296aeca

[16] Ionita, C.C., Siddiqui, A.H., Levy, E.I., Hopkins, L.N., Snyder, K.V. and Gibbons, K.J. (2011) Acute Ischemic Stroke and Infections. Journal of Stroke and Cerebrovascular Diseases, 20, 1-9. https://doi.org/10.1016/j.jstrokecerebrovasdis.2009.09.011

[17] Jin, R., Liu, L., Zhang, S., Nanda, A. and Li, G. (2013) Role of Inflammation and Its Mediators in Acute Ischemic Stroke. Journal of Cardiovascular Translational Research, 6, 834-851. https://doi.org/10.1007/s12265-013-9508-6 